\title{
DEPREDACIÓN DE PSELLIOPUS LATISPINA (HEMIPTERA: REDUVIIDAE) SOBRE TETRANYCHUS URTICAE (ACARI: TETRANYCHIDAE)
}

\author{
Salvador ORDAZ-SILVA, ${ }^{1}$ Julio C. CHACÓN-HERNÁNDEZ, AguSTín \\ HERNÁNDEZ-JUÁREZ, MELCHOR CEPEDA-SILLER, GABRIEL \\ GALLEGOS-MORALES Y JERÓNIMO LANDEROS-FLORES
}

Departamento de Parasitología Agrícola, Universidad Autónoma Agraria Antonio Narro, Calzada Antonio Narro 1923, C.P. 25315. Buenavista, Saltillo, Coahuila, México,

$1<$ ordaz_silva@hotmail.com>

Ordaz-Silva, S., Chacón-Hernández, J. C., Hernández-Juárez, A., Cepeda-Siller, M., GallegosMorales, G. y Landeros-Flores, J. 2014. Depredación de Pselliopus latispina (Hemiptera: Reduviidae) sobre Tetranychus urticae (Acari: Tetranychidae). Acta Zoológica Mexicana (n.s.), 30(3): 500-507.

RESUMEN. Se reporta la capacidad depredadora de Pselliopus latispina Hussey (Hemiptera: Reduviidae) sobre el ácaro de dos manchas Tetranychus urticae Koch (Acari: Tetranychidae), bajo condiciones de laboratorio. Se utilizaron ninfas de P. latispina de primer instar, provenientes de masas de huevos recolectadas en la Sierra de Álvarez, San Luis Potosí, sobre árboles de cedro blanco Juniperus flaccida Schltdl. (Cupressaceae) y hembras adultas de T. urticae desarrolladas en plantas de frijol Phaseolus vulgaris L. (Fabaceae), originadas a partir de recolectas en huertas de manzana Malus domestica Borkh. (Rosaceae) de Huachichil, Arteaga; Coahuila. Se colocaron ninfas individuales de P. latispina con 50 hembras adultas de T. urticae en cajas Petri, con la finalidad de observar el comportamiento depredador de las ninfas sobre el ácaro. Los datos de depredación fueron analizados con ANOVA y separación de medias mediante pruebas de rango múltiple de Tukey. Se observó la depredación a las 8, 16 y 24 horas y se contabilizó la mortalidad de ácaros. La depredación de P. latispina se incrementó proporcionalmente con el tiempo de exposición, con un consumo promedio de 20, 37 y 48 ácaros a las 8, 16 y 24 horas respectivamente, brindando una nueva opción de manejo del ácaro de dos manchas.

Palabras clave: Chinche asesina, ácaro de dos manchas, control biológico.

Ordaz-Silva, S., Chacón-Hernández, J. C., Hernández-Juárez, A., Cepeda-Siller, M., GallegosMorales, G. \& Landeros-Flores, J. 2014. Predation of Pselliopus latispina (Hemiptera: Reduviidae) on Tetranychus urticae (Acari: Tetranychidae). Acta Zoológica Mexicana (n.s.), 30(3): 500-507.

ABSTRACT. The predatory capacity of Pselliopus latispina Hussey (Hemiptera: Reduviidae) on two spotted mite Tetranychus urticae Koch (Acari: Tetranychidae) under laboratory conditions is reported. Nymphs of $P$. latispina first instar were used, from egg masses collected in the Sierra de Alvarez, San

Recibido: 10/10/2013; aceptado: 10/09/2014 
Luis Potosi, on junipers trees Juniperus flaccida Schltdl. (Cupressaceae) and adult females of T. urticae developed these in bean plants Phaseolus vulgaris L. (Fabaceae), originated from collections of Huachichil, Arteaga; Coahuila on apple orchards Malus domestica Borkh. (Rosaceae). Individual nymphs of $P$. latispina were placed with 50 adult females of T. urticae in Petri dishes; this later was done in order to observe feeding behavior of nymphs on mites. Predation data were analyzed by ANOVA and mean separation by Tukey's multiple range. After 8, 16 and 24 hours, predation was observed, and mite mortality was quantified. Predation of $P$. latispina increased proportionally with the exposure time, with an average consumption of 20,37, and 48 mites at 8,16 , and 24 hours respectively, this fact provided a new option for managing two spotted mite pest.

Key words: Assassin bug, two spotted mite, biological control.

\section{INTRODUCCIÓN}

El control de plagas por medio de sus enemigos naturales (depredadores, parasitoides y patógenos), conocido como control biológico, constituye uno de los componentes principales del manejo integrado de plagas. La adaptación de esta estrategia ayuda a reducir el uso de insecticidas, lo que hace al control de plagas más económico, ecológico y socialmente aceptable (De Bach \& Hagen 1991); sin embargo, existe poca información sobre el uso de diversos enemigos naturales como reguladores de poblaciones plaga, pero existe un interés en el descubrimiento de depredadores generalistas que no sean denso-dependientes para la búsqueda de su presa (Murdoch et al. 1985).

La familia Reduviidae, un grupo del orden Hemiptera (Insecta), con más de 6000 especies descritas (Forero et al. 2008), presenta principalmente especies depredadoras generalistas que se alimentan de una amplia variedad de presas como insectos y otros artrópodos terrestres (Miller 1971). Para obtener alimento, estos insectos, conocidos comúnmente como chinches asesinas, se posan sobre la planta a la espera de su presa, a la cual capturan con sus patas anteriores y paralizan inmediatamente insertándoles el pico (Brailovsky et al. 2007). La acción depredadora de este grupo se ha registrado en más de 18 especies de insectos plaga de los órdenes Lepidoptera, Coleoptera y Hemiptera, tanto en situaciones de campo como de laboratorio (Ambrose 1999, Sahayaraj 2003, 2006).

Aunque los redúvidos son poco específicos en la selección de su presa, podrían ser de utilidad como agentes de control biológico en programas de manejo integrado de plagas (Sahayaraj 2004, 2006) y contribuir de esta manera para reducir los daños causados por especies plaga, incluyendo ácaros fitófagos (Sahayaraj \& Sujatha 2011) como el ácaro de dos manchas Tetranychus urticae, plaga muy importante en hortalizas y otros cultivos de importancia económica (Opit et al. 2004, Liburd et al. 2007).

La familia Reduviidae incluye al género Pselliopus, un grupo depredador de insectos y otros artrópodos, que se caracteriza por tener en el hemiélitro una areola cuadrangular, artejo rostral I más corto que el II y el fémur anterior más corto que el posterior (Brailovsky \& Barrera 2004). El género comprende 27 especies descritas, 
distribuidas desde Canadá hasta América del Sur, de las cuales 21 se registran para México (Brailovsky \& Barrera 2004, Mariño-Pérez 2006, Brailovsky et al. 2007). Este género podría desempeñar un papel importante en el control biológico natural de ácaros plaga, desafortunadamente su eficiencia depredadora sobre ácaros fitófagos no ha sido investigada. Por lo anterior, el objetivo del trabajo fue determinar la capacidad depredadora de ninfas de primer instar de Pselliopus latispina sobre hembras adultas del ácaro de dos manchas T. urticae, bajo condiciones de laboratorio.

\section{MATERIALES Y MÉTODOS}

La investigación se realizó en octubre de 2012 en el área de cámaras bioclimáticas del Departamento de Parasitología Agrícola de la Universidad Autónoma Agraria Antonio Narro (UAAAN) en Buenavista, Saltillo, Coahuila, México. Las especies de insectos que se utilizaron para el estudio fueron $T$. urticae como presa y P. latispina como depredador.

La población de $T$. urticae se inició con material biológico recolectado en huertas de manzano (Malus domestica), en Huachichil, Arteaga, Coahuila, ubicado a 2511'39.6' ' $\mathrm{N}$ y $100^{\circ} 46^{\prime} 20.3^{\prime \prime}$ ' O, a una altura de $2020 \mathrm{~m}$. Los ácaros fueron colocados en plantas de frijol (Phaseolus vulgaris) para incrementar su densidad poblacional bajo condiciones controladas de laboratorio, con una temperatura de $27 \pm 2^{\circ} \mathrm{C}$ y $60 \pm 10 \%$ HR y para la realización de los bioensayos se utilizaron hembras adultas recién emergidas.

Los especímenes del ácaro de dos manchas fueron identificados a género mediante las claves taxonómicas de Krantz (1971) y a nivel de especie con las claves de Tuttle \& Baker (1968).

La población de $P$. latispina se obtuvo recolectando masas de huevos (Fig. 1) en campo, en árboles naturales de cedro blanco Juniperus flaccida en la Sierra de Álvarez, San Luis Potosí, ubicada a 22¹1'53'’ N, 100³7’03'’ O y 1840 m. Para los bioensayos se utilizaron ninfas de primer instar recién emergidas. Las ninfas se llevaron al estado adulto mediante alimentación de las mismas con ácaros de la cría de laboratorio para su identificación.

Los especímenes adultos de la chinche se identificaron a género mediante las claves de Slater \& Baranowski (1978) y para identificar la especie de Pselliopus se utilizaron las claves de Brailovsky et al. (2007) y posteriormente fue corroborada por el Dr. Luis Cervantes Peredo del Instituto de Ecología A. C. (INECOL) de Xalapa, Veracruz.

Se utilizó un diseño experimental completamente al azar con tres tratamientos y 10 repeticiones. Los tratamientos consistieron en determinar la capacidad depredadora de P. latispina sobre T. urticae en lapsos de tiempo de 8, 16 y 24 horas. Para ello, mediante un pincel de pelo de camello 000 humedecido, se colocaron 50 hembras adultas de $T$. urticae y una ninfa de $P$. latispina en una caja Petri con diámetro de 5.5 


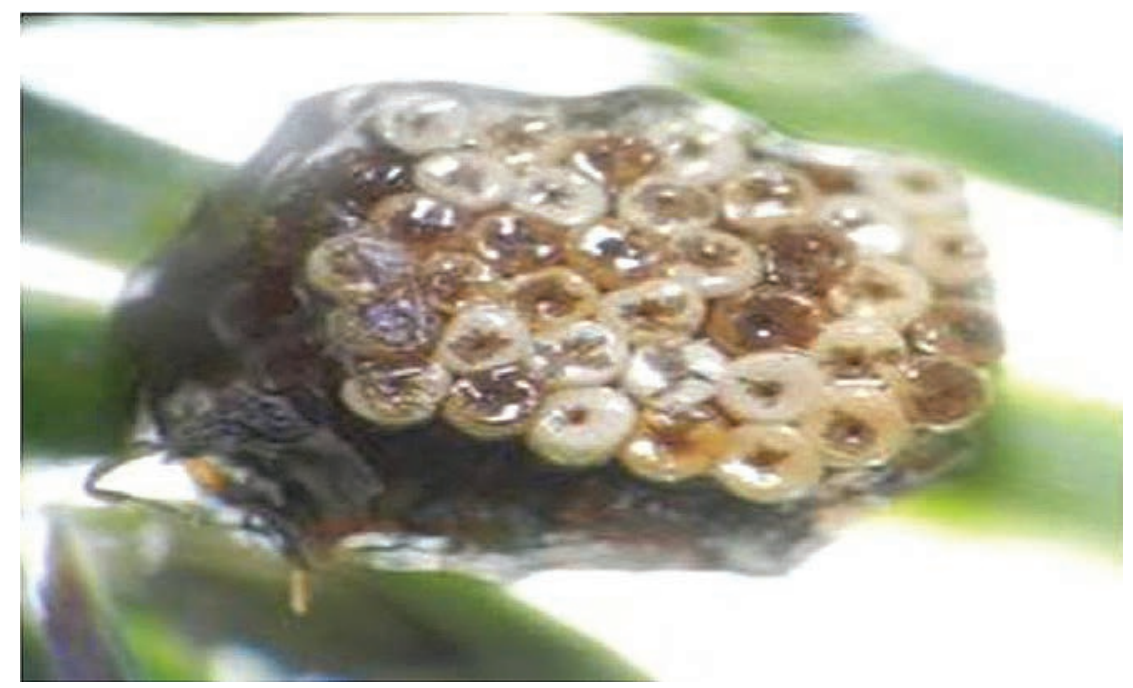

Figura 1. Masas de huevos de Pselliopus latispina colectados en campo.

cm, la cual quedó cubierta con kleen pack. El experimento se realizó bajo condiciones controladas y las observaciones se hicieron con el apoyo de un microscopio estereoscopio Carl Zeiss.

La eficiencia de búsqueda y depredación (E) se determinó mediante la ecuación:

$$
\mathrm{E}=\mathrm{Na} / \mathrm{No}
$$

Donde: Na fue el número de presas consumidas por tiempo de evaluación y No fue el número de presas ofrecidas al depredador al inicio del experimento.

Los datos de depredación se sometieron a un análisis de varianza (ANVA) y separación de medias, mediante prueba de rango múltiple de Tukey $(\alpha=0.05)$, utilizando el software SAS (SAS Institute 2002).

\section{RESULTADOS}

El análisis de varianza arrojó diferencias significativas con respecto al tiempo observado (8, 16 y 24 horas) $(F=119.09$, gl=2,29, $\mathrm{P}<0.0001)$. El número de ácaros consumidos por $P$. latispina se incrementó proporcionalmente con el tiempo de exposición, con un consumo promedio de 20, 37 y 48 individuos a las 8, 16 y 24 horas, respectivamente (Fig. 2)

Los resultados de la depredación de $P$. latispina aumentaron en todo el estudio en un $143 \%$ (8-24 horas). En el intervalo de 8-16 horas el incremento fue de $88 \%$ y de 16-24 h en $29 \%$, debido al tiempo de exposición de la plaga frente al depredador; en 


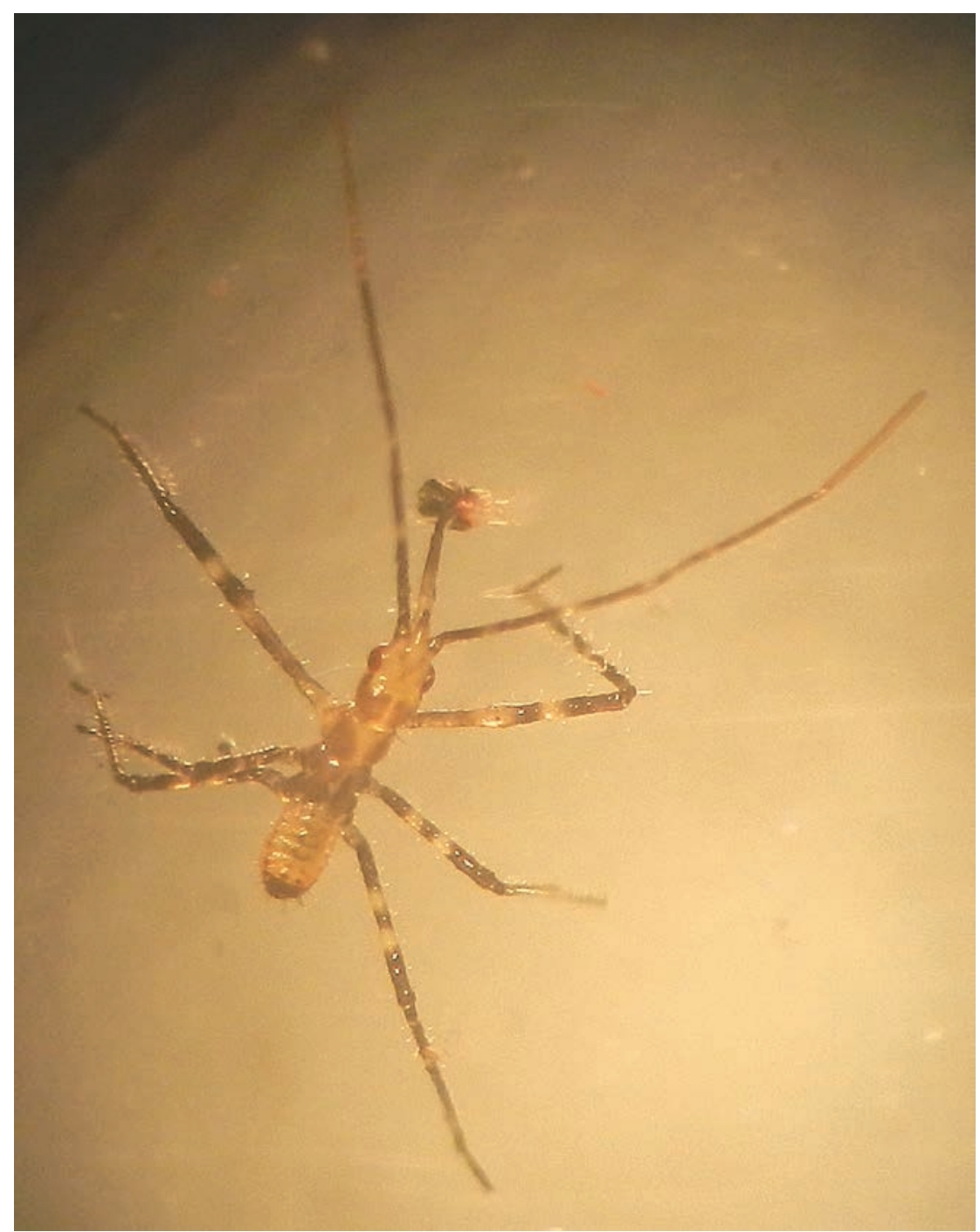

Figura 2. Ninfa de primer instar de Pselliopus latispina depredando T. urticae.

las últimas horas del experimento se refleja la saciedad del mismo, ya que el consumo de presas disminuyó en las últimas 8 horas (16-24 horas) en comparación con el intervalo de 0-8 horas (11 y 20 ácaros en promedio, respectivamente); asimismo, la eficiencia de búsqueda fue cercana a 1 transcurridas las 24 horas, lo que significó que el depredador tiene una alta capacidad de búsqueda para la localizar y consumir a su presa (Cuadro 1). 
Cuadro 1. Eficiencia de búsqueda y depredación de $P$. latispina sobre hembras de $T$. urticae.

\begin{tabular}{|c|c|c|c|c|c|c|c|}
\hline \multirow[t]{2}{*}{$\mathrm{UM}$} & \multirow[t]{2}{*}{ No } & & \multicolumn{5}{|c|}{ (Na) Presas consumidas } \\
\hline & & & $8 \mathrm{~h}$ & & $16 \mathrm{~h}$ & & $24 \mathrm{~h}$ \\
\hline 1 & 50 & & 27 & & 35 & & 49 \\
\hline 2 & 50 & & 24 & & 33 & & 50 \\
\hline 3 & 50 & & 23 & & 36 & & 42 \\
\hline 4 & 50 & & 10 & & 38 & & 50 \\
\hline 5 & 50 & & 21 & & 42 & & 48 \\
\hline 6 & 50 & & 27 & & 39 & & 49 \\
\hline 7 & 50 & & 15 & & 37 & & 48 \\
\hline 8 & 50 & & 19 & & 32 & & 50 \\
\hline 9 & 50 & & 14 & & 38 & & 50 \\
\hline 10 & 50 & & 19 & & 44 & & 47 \\
\hline Total & 500 & & 199 & & 374 & & 483 \\
\hline$\mu$ & & & 19.9 c & & $37.4 \mathrm{~b}$ & & $48.3 \mathrm{a}$ \\
\hline I & & $143.00 \%$ & & $88.00 \%$ & & $29.00 \%$ & \\
\hline $\mathrm{E}$ & & & 0.398 & & 0.748 & & 0.966 \\
\hline
\end{tabular}

$\mathrm{UM}=$ unidad muestral, $\mathrm{No}=$ Presas ofrecidas, $\mathrm{h}=$ horas; $\mu$ = Media de la depredación, $\mathrm{I}=$ Incremento de consumo, $\mathrm{E}=$ Eficiencia de búsqueda y depredación. Medias en cada columna seguidos por letras diferentes son significativamente diferentes (Tukey, $\mathrm{p} \leq 0.05$ ).

\section{DISCUSIÓN}

Esta investigación permitió registrar que P. latispina tiene la capacidad de alimentarse de ácaros de $T$. urticae, con un consumo en promedio de 48.3 ácaros en 24 horas, que corresponde al $96.6 \%$ de las presas ofrecidas. Estas observaciones concuerdan con lo mencionado por otros autores que indicaron que la mayoría de los redúvidos son depredadores de insectos y otros artrópodos terrestres (Brailovsky \& Barrera 2004, Ambrose \& Sahayaraj 1993, Sahayaraj \& Ambrose 1994, Sahayaraj 1995, Cogni et al. 2002), destacando que en los primeros instares prefieren presas de tamaño pequeño y al desarrollarse y aumentar su tamaño cambian sus preferencias a presas más grandes (Claver et al. 2002).

Estos resultados aportan información concerniente a la eficiencia depredadora de $P$. latispina durante las etapas tempranas de su ciclo de vida. El trabajo fue importante ya que no hay estudios previos de depredación de alguna especie de este género, aunque se sabe que la mayoría de los integrantes de la familia Reduviidae se caracterizan por ser depredadores generalistas. Vale la pena resaltar que $P$. latispina puede ser importante en el control biológico del ácaro de dos manchas T. urticae, lo que da la pauta para la realización de trabajos posteriores con esta y otras especies del género Pselliopus. 
En este trabajo sólo se alimentó al depredador con T. urticae, es decir, sin darle la oportunidad de preferencia hacia alguna otra presa, tal como sucede con otras especies del orden Hemiptera, como es el caso de Macrolophus caliginosus Wagner (Hemiptera: Miridae) que es un excelente depredador de T. urticae (Foglar et al. 1990); sin embargo, en evaluaciones de preferencia depredadora con ácaros T. urticae e insectos como pulgones Myzus persicae Sulzer (Hemiptera: Aphididae), se demostró que este depredador tiene mayor preferencia por los insectos (Fauvel et al. 1987).

El descubrimiento de la capacidad de P. latispina de alimentarse de T. urticae, ofrece una opción de manejo del ácaro de dos manchas como un agente de control biológico, aunque también abre la puerta para posteriores estudios de depredación sobre esta y otras especies plaga de importancia económica.

AGRADECIMIENTOS. Agradecemos al Sr. Francisco Saldaña, dueño de los terrenos donde se colectaron las masas de huevos por permitir realizar las colectas y muestreos en la zona de la Sierra de Álvarez en San Luis Potosí. Asimismo se agradece el apoyo del Doctor Luis Cervantes Peredo por la corroboración de la especie utilizada en el presente estudio.

\section{LITERATURA CITADA}

Ambrose, D. P. 1999. Assassin Bugs. Science publishers. Inc. Enfield New Hampshire: USA. 337 p. Ambrose, D. P. \& Sahayaraj, K. 1993. Predatory potential and stage preference of reduviid predator, Allaeocranum quadrisignatum (Reuter) on Dysdercus cingulatus Fabricius. Journal Biological Control, 7: 12-14.

Brailovsky, H. \& Barrera, E. 2004. Especies nuevas de Pselliopus (Hemiptera: Heteroptera: Reduviidae: Harpactorinae) de México. Anales del Instituto de Biología, Universidad Nacional Autónoma de México, Serie Zoología, 75: 313-330.

Brailovsky, Mariño R., H. \& Barrera, E. 2007. Cinco especies nuevas de Pselliopus (Hemiptera: Heteroptera: Reduviidae: Harpactorinae: Harpactorini) para México. Revista Mexicana de Biodiversidad, 78: 85-98.

Claver, M. A., Ramasubbu, G., Ravichandran, B. \& Ambrose, D. P. 2002. Searching behaviour and functional response of Rhynocoris longifrons (Stål) (Heteroptera: Reduviidae), a key predator of pod sucking bug, Clavigralla gibbosa Spinola. Entomon, 27: 339-346.

Cogni, R., Freitas, A. V. L. \& Amaral Filho, B. F. 2002. Influence of prey size on predation success by Zelus longipes L. (Heteroptera: Reduviidae). Journal of Applied Entomology, 126: 74-78.

De Bach, P. \& Hagen, D. 1991. Biological control by natural enemies. $2^{\text {nd }}$ ed. Cambridge University Press, Cambridge. 325 pp.

Fauvel, G., Malausa, J. C. \& Kaspar, B. 1987. Etude en laboratoire des principales caracteristiques biologiques de Macrolophus caliginosus (Heteroptera: Miridae). Entomophaga, 32: 529-543.

Foglar H., Malausa, J. C. \& Wajnberg, E. 1990. The functional response and preference of Macrolophus caliginosus (Heteroptera: Miridae) for two of its prey: Myzus persicae and Tetranychus urticae. Entomophaga, 35: 465-474

Forero D., Gil Santana, H. R. \& Doesburg, Van P. H. 2008. Redescription of the Neotropical genus Aristathlus (Heteroptera, Reduviidae, Harpactorinae). Pp: 85-103. In: Grozeva, S. \& N. Simov (Eds.). Advances in Heteroptera research: festschrift in honor of $80^{\text {th }}$ anniversary of Michail Josifov. 
Krantz, G. W. 1971. A Manual of Acarology. Published by O.S.U. Book Stores, Inc. Corvallis, Oregon, U.S.A. 335 pp.

Liburd, O. E, White, J. C., Rhodes, E. M. \& Browdy, A. A. 2007. The residual and direct effects of reduced-risk and conventional miticides on two-spotted spider mites, Tetranychus urticae (Acari: Tetranychidae), and predatory mites (Acari: Phytoseiidae). Florida Entomologist, 90: 249-257.

Mariño-Pérez, R. 2006. Revisión del género Pselliopus para México (Hemiptera: Heteroptera: Reduviidae: Harpactorinae). Tesis de Licenciatura, Facultad de Ciencias, Universidad Nacional Autónoma de México, México, D.F.

Miller, N. C. E. 1971. The Biology of Heteroptera. $2^{\text {nd }}$ Ed. E. W. Casey Ltd. England. 206 pp.

Murdoch, W. W., Chesson, J. \& Chesson, P. L. 1985. Biological control in theory and practice. The American Naturalist, 125: 344-366.

Opit, G. P., Nechols, J. R. \& Margolies, D. C. 2004. Biological control of twospotted spider mite, Tetranychus urticae Koch (Acari: Tetranychidae), using Phytoseiulus persimilis Athias-Henriot (Acari: Phytoseiidae) on ivy geranium: assessment of predator release ratios. Biological Control, 29: 445-452.

Sahayaraj, K. 1995. Bioefficacy and prey size suitability of Rhynocoris marginatus Fab. to Helicoverpa armigera Hubner of groundnut. Fresenius Environmental Bulletin, 4: 270-278.

Sahayaraj, K. 2003. Hunter reduviids in cotton bug control. Acrobiose, 1: 9-11.

Sahayaraj, K. 2004. Indian Insect Predators in Biological control. Daya Publishing House A Division of Astral International (P) Ltd. 352 p.

Sahayaraj, K. 2006. Ecological adaptive featurs of Hunter Reduviids (Heteroptera: Reduviidae: Reduviinae) and their biological control. Pp. 22-49. In: Guptha, V. K. A. K. \& Verma (Eds). Perspective in animal ecology and reproduction. (Vol. 3). Daya Publishing House A Division of Astral International (P) Ltd.

Sahayaraj, K. \& Ambrose, D. P. 1994. Stage and host preference and functional response of a Reduviid predator Acanthspis pedestris Stal to four cotton pests. Journal of Biological Control, 8: 23-26.

Sahayaraj, K. \& Sujatha, S. 2011. Temperature-Dependent Biology \& Physiology Reduviids. Nova Science Publishers Inc. 146 pp.

S. A. S. Institute. 2002. The SAS System for Windows, Release 9.0. SAS, Institute, Cary N. C. U. S. A.

Slater, J. A \& Baranowski, R. M. 1978. How to know the true bugs (Hemiptera-Heteroptera). The pictured key Nature Series, Wm. C. Brown Company Publishers. 256 p.

Tuttle, D. M. \& Baker, E. W. 1968. Spider Mites of Southwestern United States and a revision of the family Tetranychidae. The University of Arizona Press. 143 pp. 